\title{
A stepwise multi-assignment spreadsheet model to select student teams
}

\author{
Bilge Gokhan Celik*, Gilbert C. F. Brunnhoeffer \\ Roger Williams University \\ *Corresponding authorE-mail: bgcelik@rwu.edu:
}

\begin{abstract}
Student projects play a big role in engineering and management education. Most projects require students to work in teams, which allow students to practice their technical knowledge, foster the development of personal leadership skills, and teach the value of team accomplishments. One of the challenges of administering team projects is to create the best team compositions that give all teams the equal chance of success while maximizing interaction between students. The instructor should strive to balance each team so the team capabilities are as even as possible. Instructors also should take into account the performance of each student throughout the semester, when recreating teams for upcoming assignments. This paper presents a stepwise optimization that continues throughout the semester as the teams evolve from one assignment to another. Results show that this method can expose students to approximately $50 \%$ of their classmates within four team assignments and reasonable team grade variance.
\end{abstract}

Keywords: Team assignment, Multi-assignment optimization, Stepwise spreadsheet optimization, Instructional decision-making, Ms. Excel solver

\section{Introduction}

Teamwork in higher education is a very common teaching tool. For example, according to a survey conducted in 2002 among several universities across the United States, $80.49 \%$ of the engineering faculty use teams for design projects, while more than half of those surveyed use teams for homework/problem activities, $70.73 \%$ for in-class activities and $35.37 \%$ for case studies [1].

Programs such as construction engineering and management rely heavily on the use of project teams in the classroom starting from courses during freshmen year all the way through capstone projects in the senior year. Team assignments allow instructors to mimic project-staffing process in the construction as well as the engineering industry and initiate students' practice of working as a part of a team. There are many reasons for instructors to integrate teamwork exercises into their classroom activities. Some of the reasons are:

- $\quad$ To prepare students for diverse workplaces

- $\quad$ To teach students how to work together. weaknesses.

To facilitate a learning environment in which they can learn and benefit from each other's strengths and

- $\quad$ To improve communication skills.

To improve social interaction and help students (specifically freshmen) to build a support network during their academic careers.

These are some of the many advantages of utilizing teamwork in the classroom. Stein and Hurd [2] state that it is crucial for instructors to teach students how to work together if we want students to be prepared to work in a multicultural, diverse workplace, to live in neighborhoods that cross ethnic and racial boundaries. Oakley et. al. [3] mention in their study that compared to a traditional learning experience, students who learn as a member of small groups achieve higher grades, learn at a deeper level, retain information longer, are less likely to drop out of school, acquire greater communication and teamwork skills, and gain a better understanding of the environment in which they will be working as professionals. According to Johnson and Johnson [4] students' ability to work within a team as a group member may be one of the most important interpersonal skills since this will influence their employability, productivity, and career success. Chapman [5] takes this statement even further and states that the ability to work with others is more than just important to many businesses when hiring employees, but mandatory. 
In addition to the pedagogical and career related research that continues to illustrate the benefits of teamwork, accreditation programs such as the ones by Accreditation Board for Engineering and Technology (ABET) for Engineering as well as American Council for Construction Education (ACCE) for construction engineering and management programs have requirements in regards to teamwork. ABET [6] lists "an ability to function on a multidisciplinary teams" as one of its required student outcomes. Similarly, ACCE [7] makes it a clear requirement that the curricula address the constructor's role as a member of a multi-disciplinary team.

Although functioning in a team is a critical skill for students it is as important for them to get to know each other and build a support network throughout their academic and professional careers. As a part of a group, students not only learn to perform their best in a team setting but also build relationships with their team members along the way. This helps in return increase the networking opportunities among students, which can be specifically helpful in programs such as executive graduate programs. However, building a support network can be a critical achievement for undergraduate students as well. Students can increase their emotional intelligence [8] by meeting with and developing quality relationships with each others, which according to Schutte et al. [9], includes their ability to understand and regulate others as well as one's own emotions. Thus, it is critical to note that this study focuses not only on how to configure optimum teams but also to reconfigure each team throughout the semester to increase the networking and interaction opportunities.

The goal of this paper is to illustrate an example spreadsheet methodology for selecting team members to maximize interaction among students throughout the semester while minimizing grade variation among teams. Authors assume that optimizing these variables will increase the quality of team outputs, which can be critical especially when and if industry members sponsor these courses. Results of this study can benefit instructors not only from engineering and management programs but those from other disciplines with similar challenges in forming teams and creating a networking habit within the classroom. The model presented in this paper is a preliminary approach and requires knowledge of MS. Excel and its Solver Add-in.

\section{Configuring teams of students}

Finelli et. al. [10] summarize that team membership can be selected by students, determined randomly, or assigned by the instructor based upon individual student characteristics. Among these options, instructor-assigned teams offer the most control over the ways in which resources are distributed among teams and result in a stronger sense of fairness [10]. In addition, a study of engineering students at the United States Military Academy, found that self-selection had negative effects on students' opinions about the course and the projects [11]. As studies such as Bacon et al. [12] found that students had some of their worst team experiences with random configuration of the teams, a bigger problem with random selection is its unpredictability of assuring diversity in skills, constraints, and personalities of the team members.

Although instructor assigned team membership has proven benefits, how to create the most effective team still remains a challenge to many instructors. These challenges include determining the objective(s) and constraints of what makes a successful team of students. Other challenges may be establishing tasks for the teams as well as determining how to monitor and evaluate them [1]. Finally it is also a challenge for instructors to utilize convenient software tools to assist them in the decision process. There have been efforts in the past to address these challenges. These efforts mostly include the use of quantitative methodologies to form teams for industrial and/or academic applications. Zakarian and Kusiak [13] utilized Analytical Hierarchical Process in order to optimize a team selection matrix with desired characteristics and engineering skills. Others such as Chen and Lin [14] later used AHP by taking into consideration different descriptors of team members such as their communication skills, knowledge, experience, personality, and flexibility. Kirkwood [15] proposed an algebraic modeling, which took into consideration students' ranked preferences of a pool of industry sponsored MBA projects.

Many of the optimization studies require instructors to have access to and/or know how to use software programs to solve their models. Kirkwood [15], for example demonstrates the use of freely available Internet optimization resources in order to address this issue. However, there have been some other studies that focused on not only the mathematical optimization of the problem but also the ease of use for instructors. For example, Layton et al. [16], similar to Bacon et al. [12], developed a web-based program (Team-Maker) that allows instructors create teams based on information that is collected over an online survey that each student takes. Team-Maker allows instructors to create a free faculty account and email the data collection survey to the students. Instructors then can determine which criteria to use and their weights to finalize their team configurations using the Team-Maker software. Although Team-Maker is a relatively easy to use and a sophisticated team configuration program, it does not take a multi-assignment and multiteam case into account thus does not address our objective to increase interaction within the students by changing their teams throughout the semester. 


\title{
3 A spreadsheet model approach
}

Spreadsheet modeling is commonly used in many daily tasks among faculty members as a tool that allows fast, easy, and customizable solutions in the classroom. Many faculty members utilize MS. Excel spreadsheet application to keep records of class rosters, grades, and simple calculations regarding students' performances. Authors understand that a complicated team building model could be defined and presented in theory and possibly be solved using sophisticated optimization software programs. However, it is critical to note that the authors decided to build a model that can be solved completely by using MS. Excel and its Solver Add-in. This decision was made since MS. Excel and Solver are the tools that many instructors have easy access to. This increases the chances of the adoptability of the presented model. Instructors who would like to use this model for their courses will have the ability to modify the presented spreadsheet to fit their own needs (i.e. different number of students, groups, assignments/projects). The model presented here assumes that the success of a team can be maximized based on the qualifications of its members. Authors assume that these qualifications are directly correlated with students' performance in each team assignment, which are introduced into the model in the form of constraints and variables.

\subsection{Defining the model}

Prior to defining the model it is important to discuss that this study introduces the spreadsheet model using a sample case for which a few initial decisions are made. These basic decisions regarding the case are:

- The number of students in the class

- The number of students in a team

- $\quad$ The number of team assignments

The model presented here assumes that a course may require more than one assignment/project that requires teamwork. In those cases where there is an opportunity for forming teams in more than one occasion, authors decided to increase the interactions between students. This means that if a course has multiple assignments that will be completed in teams, each team will have at least one person different in them each time a new team is formed. The idea behind this derives from authors teaching a freshmen course in which the students barely know each other. Rotation of students from one team to another allows them to interact with each other at most and to build their support system in the program. It also allows students to be exposed to a different team dynamic every time there is a new assignment. The second decision is regarding the number of students in a team. Although every instructor has different constraints when it comes to number of students on a team, there is little research available on the optimum number of students on a team. Oakley et al. [3] recommend three to four-person teams after stating that:

\begin{abstract}
...with only two people on a team, there may not be a sufficient variety of ideas, skills, and approaches to problem solving for the full benefits of group work to be realized. Also, conflict resolution can be problematic in a pair: whether right or wrong, the dominant partner will win most arguments. On the other hand, if a team has more than five members, at least one is likely to be relatively passive unless the project is a large one that has six or more distinct roles, which few team assignments do. Many cooperative learning authorities believe that five-member teams are likely to experience the same problem. (p. 12)
\end{abstract}

This paper illustrates a spreadsheet model that is built for:

20 students

5 teams of four persons each

4 assignments within the semester that requires teams.

In order to define the model, it is important to identify the objective, variables, and the constraints of the problem. The objectives of this model are to:

Maximize the number of interactions each student has with other students

Minimize the variance among the average grade point average (GPA) of team members.

The GPA is defined as the most up to date course grade average at the point of a team assignment. This means that each assignment that is graded increases or decreases the GPA of a student as the semester progresses. Also a peer evaluation technique allows team members to evaluate their peers thus the grade of each team member has a potential to be different due to the consideration of peer evaluation. For example, if Student A is teamed up with Student B and C, by the end of the assignment, team will be assigned a grade. However, that grade will be adjusted for Students A, B, and C based on their peer evaluations. In short, although a team may receive $100 \%$ for their work each student on the team may receive less than that if their peer evaluations are low. Figure 1 illustrates an example of peer evaluation using an excel spreadsheet filled out individually by team members. This allows an individuality of the evaluation and makes the model more dynamic as the course GPA of each student changes after each assignment is graded. Decision variables of 
the model are simple. Binary variables will be assigned to each student in a matrix format indicating whether they are chosen for a certain team or not. Constraints of the project are:

Binary constraint for decision variables

Number of students on a team equals four

\begin{tabular}{|c|c|c|c|c|c|c|}
\hline & $\mathrm{C9}$ & $x<$ & $=\mathrm{IF}(\mathrm{C}$ & $S B 3>\$ B 3$, & $33, \mathrm{C} 8 * 5 \mathrm{~B} 3$ & \\
\hline 1 & A & B & C & D & $\mathbf{E}$ & $\mathbf{F}$ \\
\hline 1 & Assignment & $\mathrm{A} 100$ & & & & \\
\hline 2 & & Team Grade & & & & \\
\hline 3 & & 10.0 & Student A & Student B & Student C & \\
\hline 4 & Captain & Student A & $10.0 \%$ & $45.0 \%$ & $45.0 \%$ & $100.0 \%$ \\
\hline 5 & & Student A & $10.0 \%$ & $45.0 \%$ & $45.0 \%$ & $100.0 \%$ \\
\hline 6 & Member 1 & Student B & $10.0 \%$ & $48.0 \%$ & $42.0 \%$ & $100.0 \%$ \\
\hline 7 & Member 2 & Student C & $10.0 \%$ & $45.0 \%$ & $45.0 \%$ & $100.0 \%$ \\
\hline 8 & & & $30.0 \%$ & $137.3 \%$ & $132.8 \%$ & \\
\hline 9 & & & 3.00 & 10.00 & 10.00 & \\
\hline 10 & Step & & & & & \\
\hline 11 & 1 & \multicolumn{3}{|c|}{ Find the row where your name is entered } & & \\
\hline 12 & 2 & \multicolumn{5}{|c|}{$\begin{array}{l}\text { Make no entry in row } 5 \text {, the values in row } 5 \text { automatically duplicate the } \\
\text { Captain votes }\end{array}$} \\
\hline 13 & 3 & \multicolumn{5}{|c|}{$\begin{array}{l}\text { In the row containing your name, enter the } \% \text { participation of each team mate } \\
\text { in columns } \mathrm{C}, \mathrm{D}, \& \mathrm{E}\end{array}$} \\
\hline 14 & 4 & \multicolumn{5}{|c|}{$\begin{array}{l}\text { Adjust your entries in columns C, D, \& E so the total in column F of your } \\
\text { row equals } 100 \% \text {. }\end{array}$} \\
\hline 15 & 5 & \multicolumn{3}{|c|}{ Submit your vote with your Homework. } & & \\
\hline
\end{tabular}

Fig. 1: Sample peer evaluation sheet for teams. Each student is asked to fill a peer evaluation file after submitting a group assignment.

\subsection{Setting up the spreadsheets}

Initial step to set up the spreadsheet is to design the initial excel tab as a grade sheet that can be used when creating teams for each assignment. Figure 2 illustrates a grade sheet with 20 students and 4 future team assignments. Assignment names are shown as A100, A200, A300, and A400. There are initially no grades listed on this sheet for any assignments as we assume they have not occurred yet. If the course does not have any prior assignments that are already graded at this point, the incoming GPA of each student can be used only for the purpose of creating the teams for the first assignment. Once the teams are determined for the first assignment, model shown here creates future teams based on the previous assignment's grades instead of the overall GPA. This approach is calculated by using the following formula (Eq. 1) in MS. Excel for Column D in Figure 2:

$=\mathrm{IF}(\mathrm{SUM}(\mathrm{F} 4: \mathrm{I} 4)=0, \mathrm{E} 4, \mathrm{SUM}(\mathrm{F} 4: \mathrm{I} 4))$

Variance column in Figure 2 is build for a specific reason. Each time an assignment is graded students' total scores in column D will be changing. Also each student's variance from the course average will be changing. Due to Excel Solver limitations and to speed up the process, the proposed model creates teams one by one at a time. Due to this, instructor needs to determine which student to first create a team for. After multiple trials and comparisons, authors decided to create teams initially for those students with the highest variation from the course average. This allows the model to choose from a larger pool of potential teammates for those students with too high or too low of grades. This eventually allows minimizing variation among the average GPA of all the teams. For this reason, the variance column is created in Figure 2 with a formula to calculate the absolute value of the difference between a student's GPA and the class average. The "Rank" column in Figure 2 is created next in order to rank each student's variance from highest to lowest. This allows instructors to identify the student(s) with the highest rank (highest variance) and to initially create a team for them to bring the team average as close to the course average as possible. According to Figure 2, instructor should start creating a team for Student $\mathrm{E}$ who has the highest variance thus ranked first.

According to Figure 2, instructor should start creating a team for Student E who has the highest variance thus ranked first. 


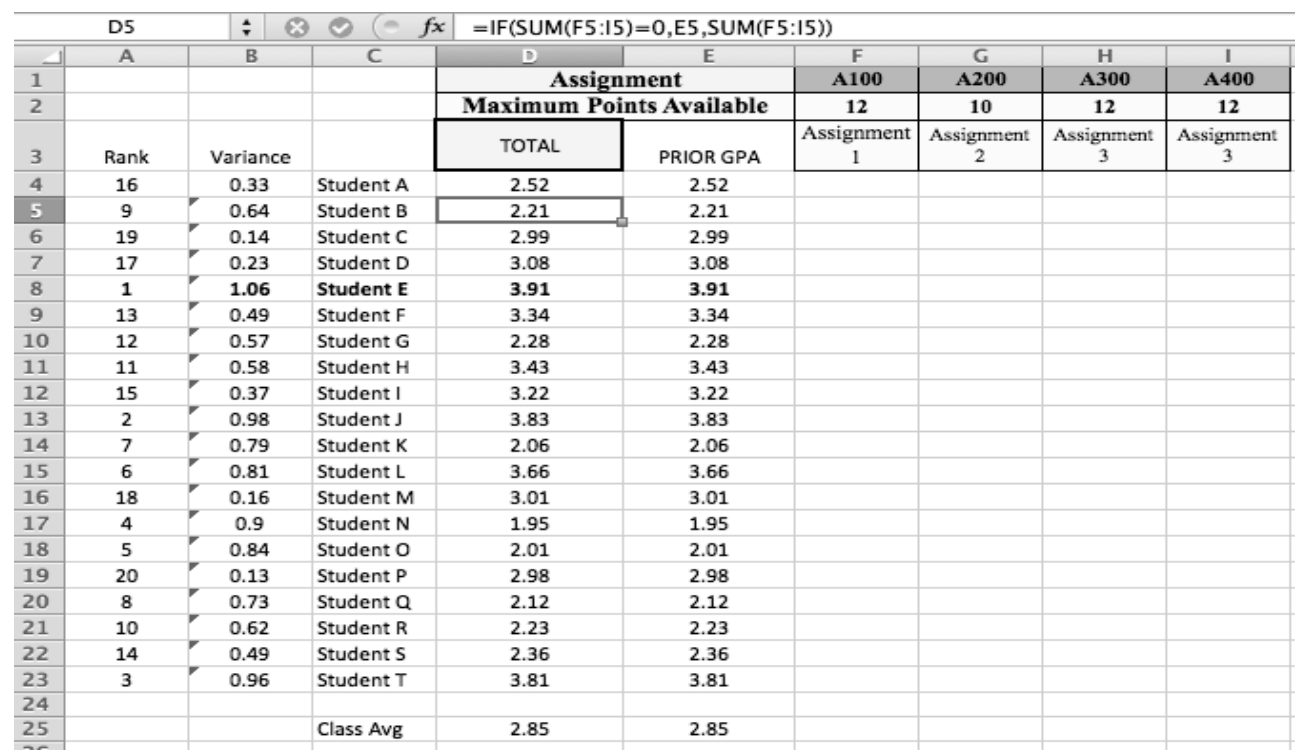

Fig. 2: Gradesheet tab illustrating students' grade variance from the average grade of the class.

The second step is to setup another tab that can be used as the master matrix in which a students' interaction with each other can be calculated. This allows the instructor to change at least one student within each new team assignment to increase the opportunity to work with different individuals. Figure 3 illustrates the master sheet structure for this purpose in which each cell has the following sample formula (Eq. 2), which allows the master matrix to continuously display a history of student's team members:

=IF('A100'!D3+'A200'!D3+'A300'!D3+'A400'!D3<100,"','A100'!D3+'A200'!D3+'A300'!D3+'A400'!D3)

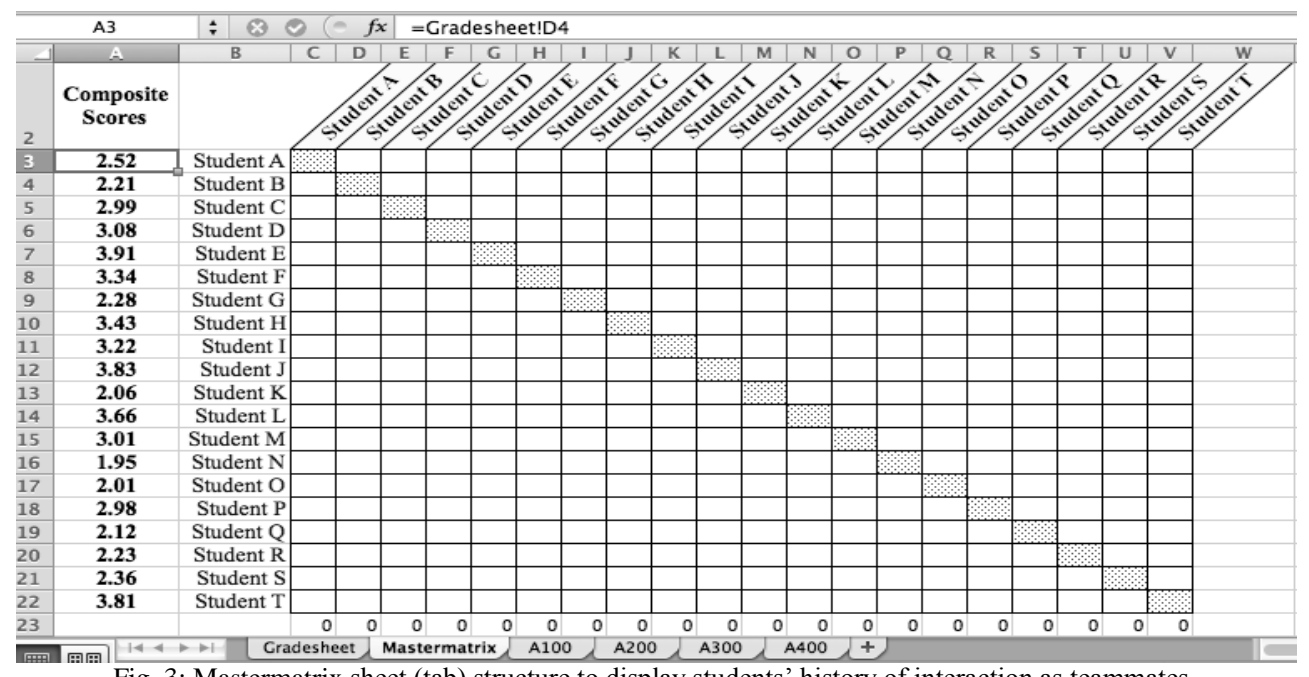

Fig. 3: Mastermatrix sheet (tab) structure to display students' history of interaction as teammates.

Next step is to replicate the matrix shown in Figure 3 for each assignment that a team needs to be built for which in this case are A100, A200, A300, and A400. A sample assignment matrix for A100 is given in Figure 4. Once the tabs are created (by copying and pasting from the mastermatrix), instructor can decide the teams for each assignment either manually or by using Excel Solver Add-in for a more precise approach. Three critical columns to mention at this point are the "Team Average", "Variance from Class", and "\# of Team Members" columns shown in Figure 4. "Team Average" column calculates the average GPA of each team. This becomes possible when Excel Solver assigns binary variables for each cell indicating the teams. For example, if the row for Student A has number 1 assigned for the columns Student I, J, and P, this means that Solver assigned those three students to a team with Student A. In that case the team average becomes the average grade thus far of those four students. Variance from class is the difference between the team's average and the class average. "\# of team members" show the number of students assigned to each team by summing the binary values in each row. By the end of the Solver optimization, each row should display 
number 4 for this case study as the authors decided to go with four-person teams. The formula (Eq. 3-5) within each of these columns are described in more detail below using Student A in Row 3 as the example:

- $\quad$ Team Average Formula (summarized using sigma function):

$$
=\frac{\left(\sum_{k=C}^{V} I F(k 3=1, \operatorname{LOOKUP}(\$ k 2, \$ B \$ 3: \$ B \$ 22, \$ A \$ 3: \$ A \$ 22), 0)\right)}{\operatorname{COUNTIF}(C 3: V 3,1)}
$$

- $\quad$ Variance from Class Formula: =ABS(X3-Gradesheet!\$D \$25)

- $\quad$ \# of Team Members Formula: =COUNTIF(C3:V3,1)

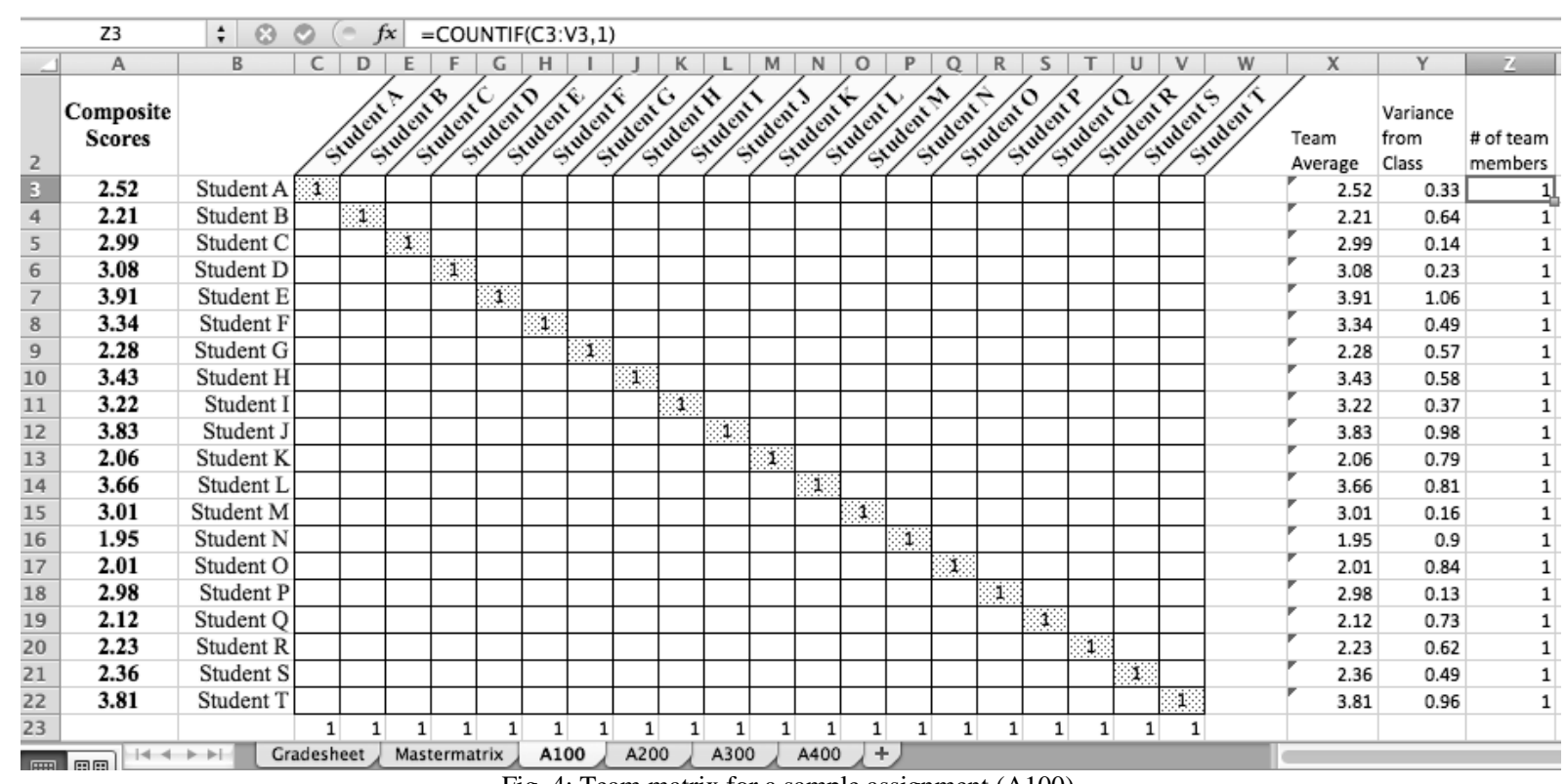

Fig. 4: Team matrix for a sample assignment (A100)

\section{Results}

Basic and free version of Excel Solver is not the best optimization tool for a team assignment problem with many binary variables [15]. That is why current literature shows that researchers shift to other more sophisticated modeling programs in order to solve similar problems. Authors of this paper decided that although those programs can create better and faster solutions, average faculty member does not have access to or the training for those programs, making the current literature difficult to utilize in the classroom. This paper illustrates a methodology to overcome the limitations of Excel Solver. The method illustrated here is a multistep optimization that decreases the number of variables by solving them at different times. This decreases the load on Excel Solver and makes the solution of a complicated binary team assignment problem possible using Excel Solver.

As explained previously, the model requires determining five teams for each assignment and one team at a time. Solver model can be configured for one of the rows in the matrix initially to determine that individual's team members. Doing this five times creates all the teams for all students. Ranking of the variance in the gradesheet tab becomes critical for this purpose. Since the instructor needs to choose a student to build a team for this methodology suggests picking the five students with highest variance, one at a time.

In our case study, the student with the highest rank (highest variance) is Student E. The initial solver model is built for Student E by determining the empty cells on Student E's row as decision variables. The "variance from class" cell on the same row is selected as the objective function to be minimized. Constraints are configured as:

- $\quad$ All variables are binary numbers

- "\# of team members" cell in Row 7 (on Student E's row) equals 4.

By choosing evolutionary model option and making sure all variables are non-negative, Solver determines the best possible team members for Student E. Figure 5 shows the results of the initial optimization attempt. 


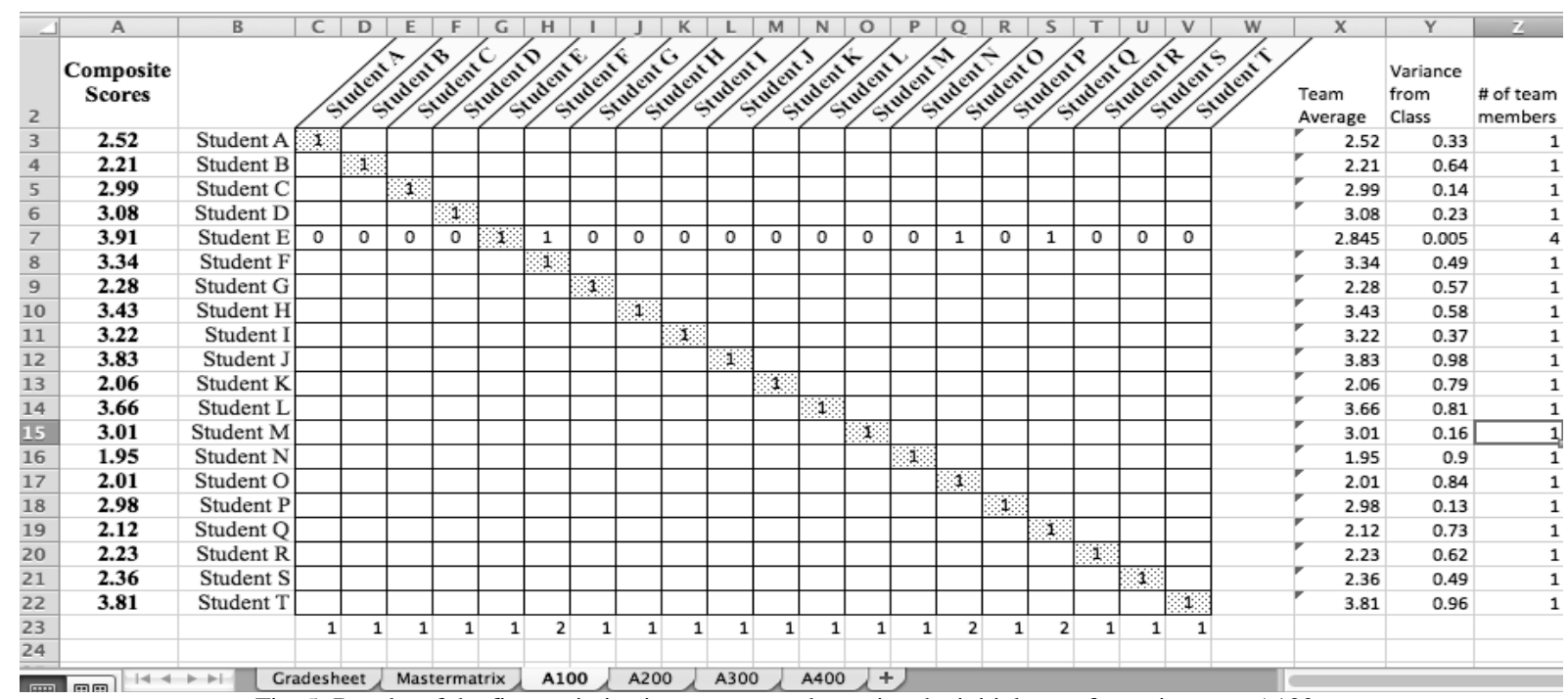

Fig. 5: Results of the first optimization attempt to determine the initial team for assignment A100.

Results show that Team 1 includes Students E, F, O and Q. Their team average variance is only $0.005(0.125 \%$ based on a maximum available grade of 4.0) from the class average so Team 1 overall is an accurate representation of the class. In order to determine the rest of the teams, instructor needs to review the rankings in the Gradesheet tab and determine the initial team member of Team 2, 3 and 4 in that order. As each team is determined, it is important to replace all the cells that Solver identified as " 0 " and " 1 " with other values to allow an accurate model for the next assignment. Cells with " 0 " should be deleted and be left blank, as those cells still are viable team members for future teams. Cells with "1" may be replaced with a high value such as "100" which can also represent A100. This allows us to visualize the teams for each assignment by locating 100 for A100, 200 for A200, etc. Highlighting those students who are already assigned to a team also helps eliminate those cells as decision variables for upcoming teams in A100 (Fig. 6). Number 100 to represent A100 is also placed on every intersection of team members on the matrix as illustrated in Figure 6.

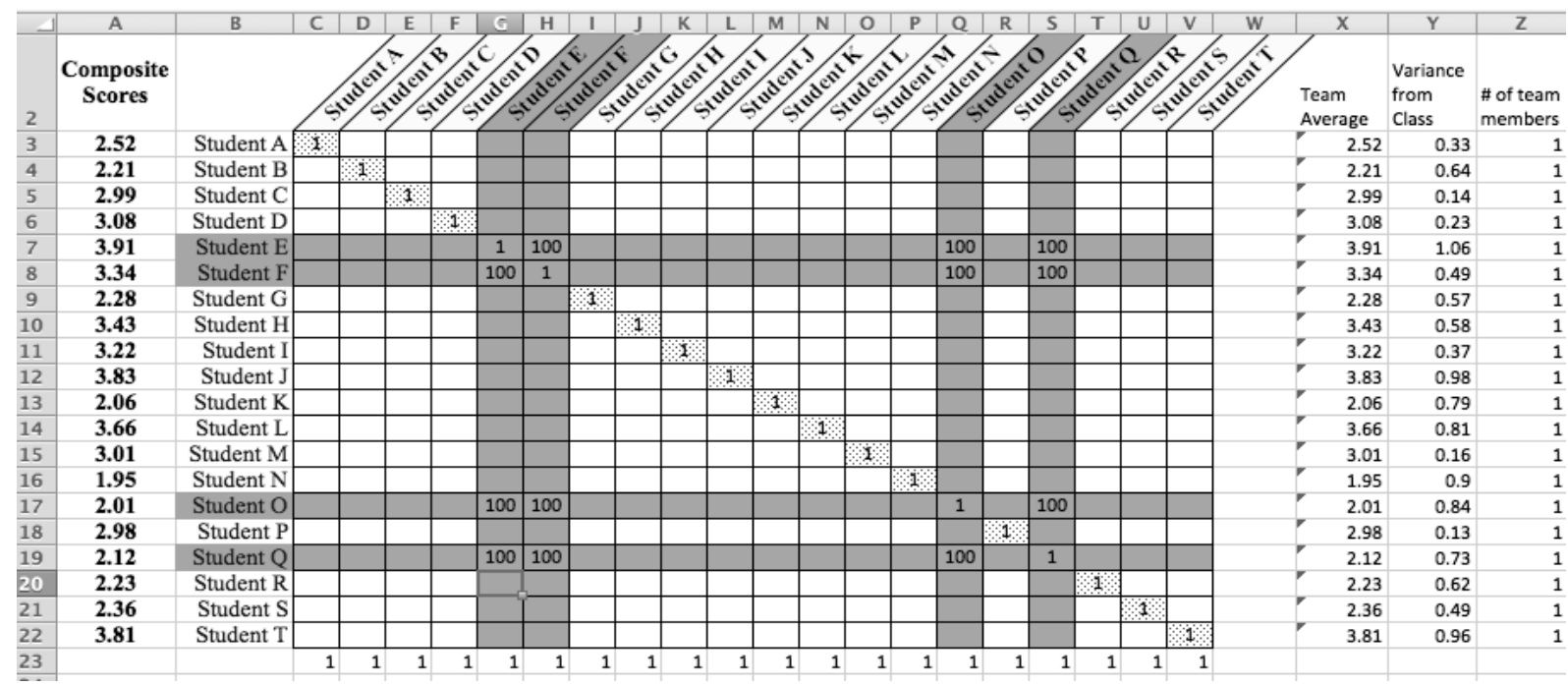

Fig. 6: Team 1 determined and highlighted on A100 tab.

The screen capture of the solver screen when calculating the second team for A100 is given in Figure 7. For Team 2, Student $\mathbf{J}$ is chosen as the row with the highest variance and decision variables as well as the constraints are modified accordingly (Fig. 7). Results of the second solver optimization are given in Figure 8. Team 2 is made of Student J, B, D, and $\mathrm{G}$ and their average variance from the class average is only $4.4 \mathrm{E}-16$. 


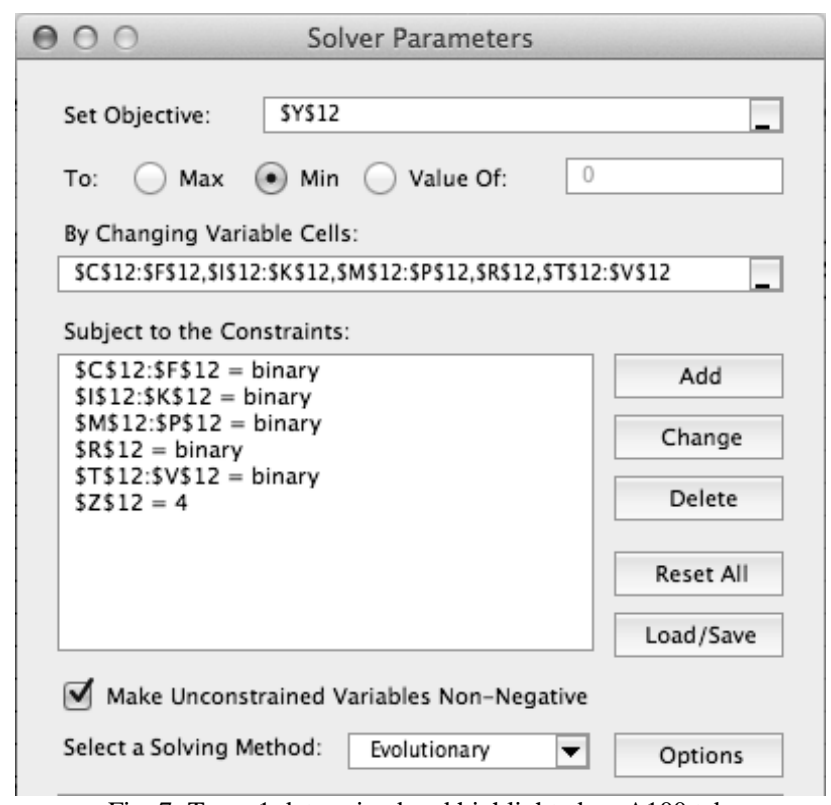

Fig. 7: Team 1 determined and highlighted on A100 tab.

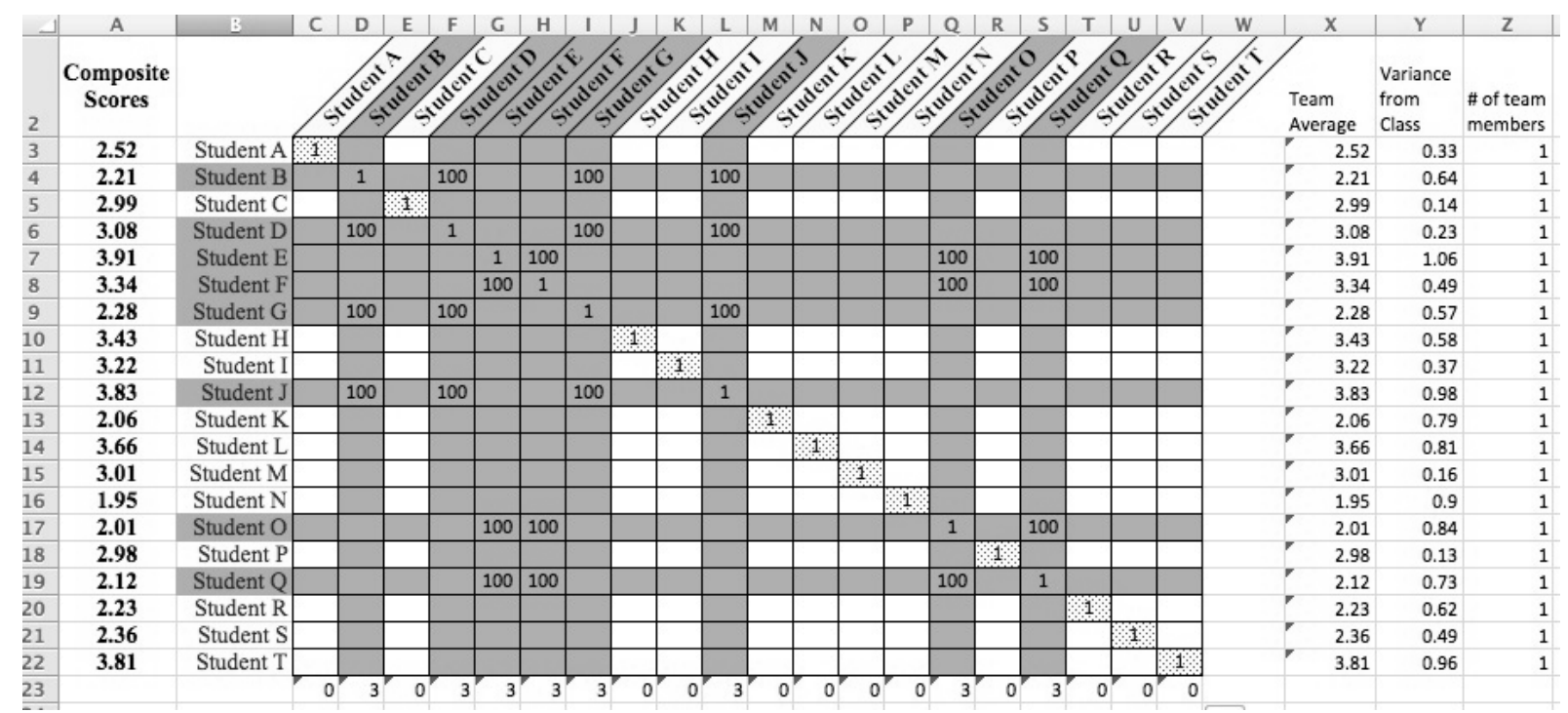

Fig. 8: Results of solver optimization for creating Team 2.

Third team for A100 starts with Student T as the student with 3rd highest GPA variance from the class average. Results are given in Figure 9. Based on the results of the optimization, Student T is teamed up with Students C, R, and S. When the average grade of students in Team 3 is compared to the overall average grade of the class, Team 3 has a GPA variance of 0.0025 . This variance is less than $0.1 \%$ of the maximum grade available (4.0), which illustrates that similar to Team 1 and 2, Team 3 also is a strong representation of the class.

Figure 9 also presents the cells that are highlighted that represent students who already are assigned to a team by the end of forming Team 3. This means that all the cells that are highlighted are potential decision variables for the next iteration of team assignment. This allows the instructor visualize the remaining students as well as avoid mistakes of assigning the same student into multiple teams.

Fourth team for A100 requires solver to optimize for one last time. All the students left after the fourth optimization, automatically form the final team. Student $\mathrm{N}$ is chosen as the 4th highest rank of GPA variance. Solver optimization determines that Team 4 should include Student N along with Students H, M, and P with a GPA variance of 0.0075 $(<0.1 \%$ of maximum available grade). Remaining students A, I, K, and L are automatically left for Team 5, which has a GPA variance of 0.015 ( $0.375 \%$ of maximum available grade). Final matrix showing all rows and columns highlighted can be seen in Figure 10. Final teams list along with their average GPAs and variance from overall course GPA is given in Table 1. 


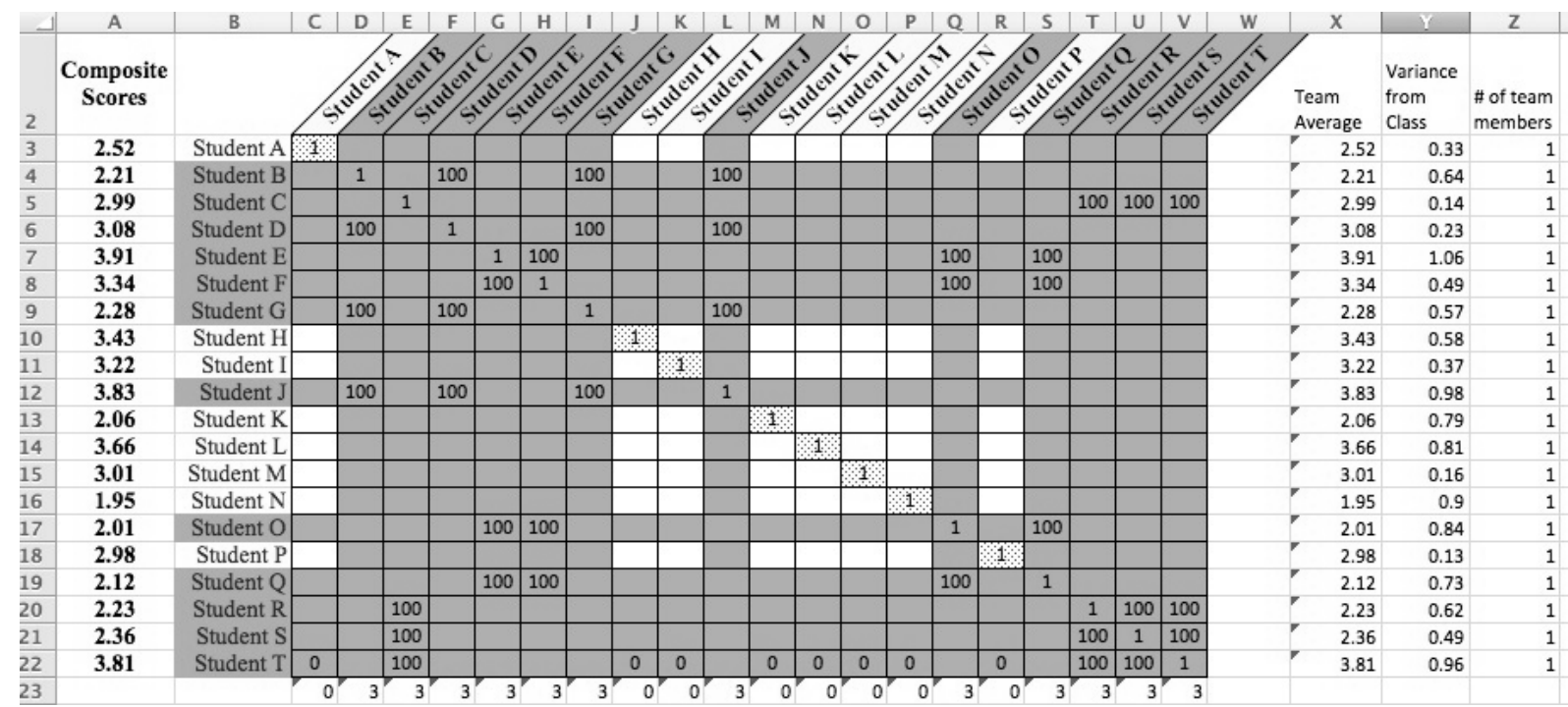

Fig. 9: Results of solver optimization for creating Team 3.

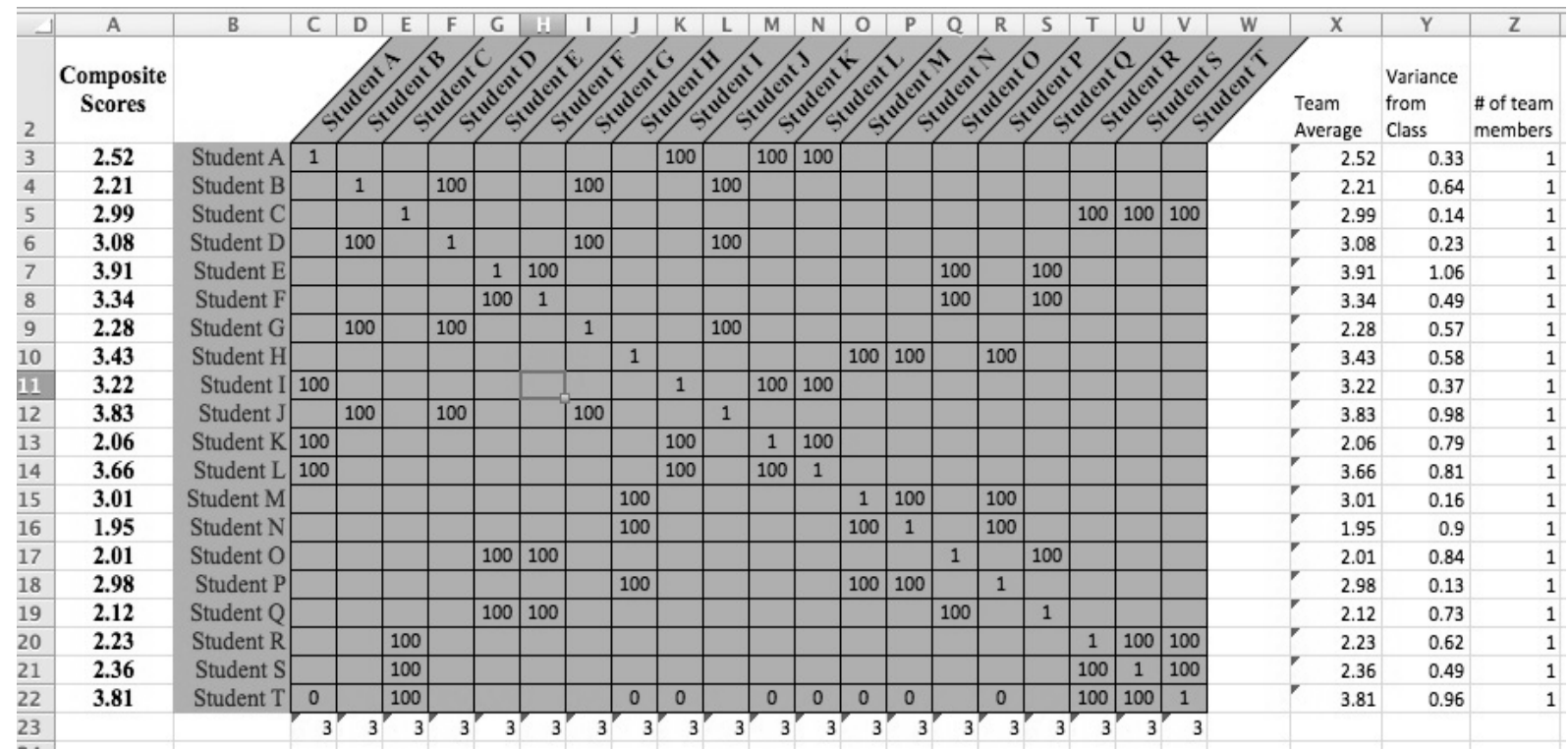

Fig. 10: All five teams are optimized and students working with each other are expressed by number 100.

Table 1: Team members, average GPAs, and variance from class GPA average.

\begin{tabular}{lccccc}
\hline A100 & Team 1 & Team 2 & Team 3 & Team 4 & Team 5 \\
\hline & Student E & Student J & Student C & Student N & Student A \\
& Student F & Student B & Student R & Student H & Student I \\
& Student O & Student D & Student S & Student M & Student K \\
& Student Q & Student G & Student T & Student P & Student L \\
\hline Team Average & 2.845 & 2.85 & 2.8475 & 2.8425 & 2.865 \\
\hline Variance & 0.005 & $<0.001$ & 0.0025 & 0.0075 & 0.0150 \\
\hline
\end{tabular}

Once the instructor is ready to form the teams for the next assignment, these final matrix cell values from the most current assignment (A100) should be copied and pasted on to the next tab (A200). This allows the users to identify which students worked with whom thus far, and eliminate those cells (with number 100) from the list of variables in the upcoming team assignments.

It is also important for the instructor to fill out the grades for the most recent assignment (A100) using the initial gradesheet tab. This enables the use of the most up to date grades, from recent assignments, as the student GPAs for the next team optimization. 


\subsection{Determining teams for upcoming assignments}

Teams for assignment A100 is already determined but if the course has multiple assignments, teams may need to change for those assignments in order to increase the interaction among students. This paper assumes there are four assignments in this course that requires teamwork. In order to determine the teams for A200, A300, and A400 steps that need to be taken is given below.

STEP 1: Copy and paste (only the values) of the matrix in the most recently completed assignment tab (A100 initially), into the upcoming optimization tab (A200 initially).

- $\quad$ STEP 2: Enter the grades from the most recent assignment (A100 initially) into the gradesheet tab.

- $\quad$ STEP 3: Complete the same steps taken for A100 by starting to utilize solver for the student with the highest variance from the course average.

- $\quad$ STEP 4: When determining decision variables in Solver, do not choose cells with any numbers in them since they indicate a group experience of the pair of students as teammates in prior assignments. This will guarantee that at least $25 \%$ of the team will be different for each assignment.

STEP 5: Return to Step 1 for A300, and A400 assignments as each assignment is graded throughout the semester. For example, copy and paste the matrix in A200 into A300 before starting team selection for the third assignment. If at any point the student selected with a high variance do not have enough empty cells as variables, proceed to the student with next highest variance.

The final results of team optimization for all teams on the A200 matrix are given in Figure 11. If needed conditional formatting can be utilized in the Mastermatrix tab to evaluate the frequency of individuals working with each other, by shading cells with the higher numbers darker, and lower numbers lighter List of all teams for A200 along with the team GPA averages and the variances from class average are given in Table 2.

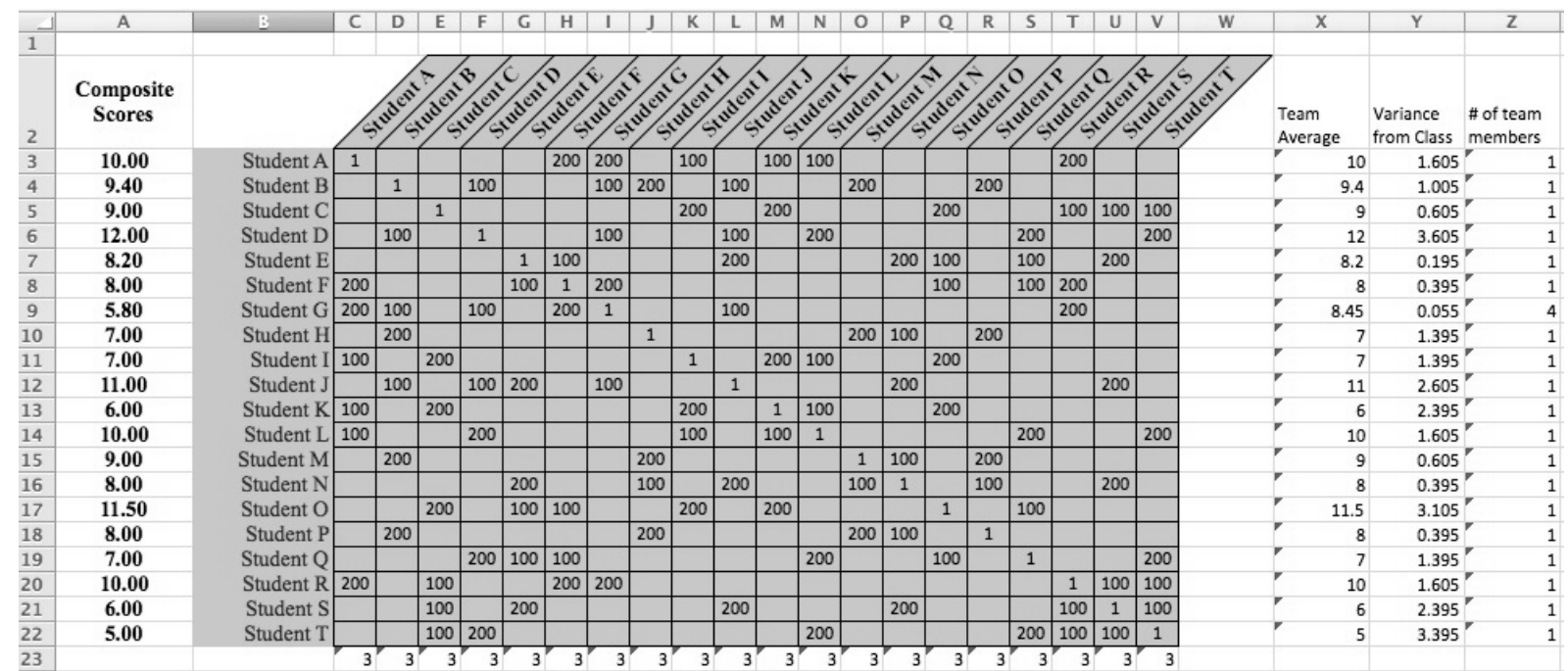

Fig. 11: All five teams for A200 are optimized and students working with each other are expressed by number 200.

Table 2: Team members, average GPAs, and variance from class GPA average for A200.

\begin{tabular}{lccccc}
\hline A200 & Team 1 & Team 2 & Team 3 & Team 4 & Team 5 \\
\hline & Student D & Student O & Student J & Student G & Student B \\
& Student L & Student C & Student E & Student A & Student H \\
& Student Q & Student I & Student N & Student F & Student M \\
& Student T & Student K & Student S & Student R & Student P \\
\hline Team Average & 8.5 & 8.38 & 8.3 & 8.45 & 8.35 \\
\hline Variance & 0.105 & 0.020 & 0.090 & 0.055 & 0.045 \\
\hline
\end{tabular}

Following the steps described earlier all teams for all assignments can be determined as the semester progresses and grades for each assignment is entered into the spreadsheet. Table 3 presents the optimum team configurations for A300 and A400 as well as the team average GPAs and variance from the class average.

Exploring the variances of Team GPAs for all four assignments, authors calculated an average variance of $0.15 \%$ for A $100,0.53 \%$ for A200, 2.77\% for A300, and 1.56\% for A400. In order to evaluate the success of the optimizations it is critical to also report on the initial grade average variances. The average variance of each student from the class mean 
GPA prior to optimization of A100 to A400 is calculated at $\% 14.78, \% 13.62, \% 5.72$, and $\% 6.21$, respectively. It is reasonable to assume that the authors were successful in team optimization by significantly reducing the grade variances of students when they are formed into teams. It should be noted that these numbers would be different for different case studies. However the method described in this study should yield the optimum (lowest) grade variation of teams using MS. Excel.

Table 3: Team members, average GPAs, and variance from class GPA average for A300.

\begin{tabular}{lccccc}
\hline A300 Teams & Team 1 & Team 2 & Team 3 & Team 4 & Team 5 \\
\hline & Student D & Student T & Student K & Student M & Student L \\
& Student I & Student A & Student B & Student C & Student P \\
& Student N & Student G & Student H & Student E & Student Q \\
& Student O & Student J & Student R & Student F & Student S \\
\hline Team Average & 24.33 & 25.23 & 24.675 & 26.375 & 25.65 \\
\hline Variance & 0.925 & 0.025 & 0.575 & 1.125 & 0.400 \\
\hline A400 Teams & Team 1 & Team 2 & Team 3 & Team 4 & Team 5 \\
\hline & Student L & Student R & Student J & Student G & Student A \\
& Student H & Student I & Student C & Student E & Student B \\
& Student M & Student P & Student F & Student N & Student D \\
& Student O & Student Q & Student K & Student S & Student T \\
\hline Team Average & 25.25 & 25.18 & 26.575 & 24.35 & 0.900 \\
\hline Variance & $<0.001$ & 0.075 & 1.325 & & 0.350 \\
\hline
\end{tabular}

Although minimizing team grade averages is one of the objectives of this model, it is important to review the results of the second objective: Maximize the number of interactions each student has with other students. Figure 12 presents a color/shade-coded exposure matrix that shows the experience of each student with their classmates. As can be seen in Figure 12, the average exposure of students is $49 \%$. This shows that using the methodology presented in this paper, in a 20-student classroom, students, on average, had the opportunity to work with approximately 10 other students (half of the class population) through four different assignments. There is not a specific area of the matrix that is dominated by a certain shade, which illustrates a homogenous and balanced distribution of each student's team experience.

\begin{tabular}{|c|c|c|c|c|c|c|c|c|c|c|c|c|c|c|c|c|c|c|c|c|c|c|c|}
\hline $\begin{array}{l}\text { Composite } \\
\text { Scores }\end{array}$ & $\begin{array}{l}\text { After A300 } \\
\text { Master }\end{array}$ & & & & & & & & & & & & & & & & & & & & & & $\begin{array}{c}\text { Exposure } \\
\text { to class } \\
\text { mates }\end{array}$ \\
\hline 27.50 & Student A & & 400 & & 400 & & 600 & 800 & & 400 & 600 & 400 & 400 & & & & & & 600 & & 700 & & $50 \%$ \\
\hline 22.90 & Student B & 400 & & & 700 & & & 400 & 800 & & 400 & 600 & & 600 & & & 600 & & 600 & & 400 & & $50 \%$ \\
\hline 25.00 & Student C & & & & & 600 & 700 & & & 600 & & 800 & & 600 & & 600 & & & 400 & 400 & 400 & & $45 \%$ \\
\hline 27.00 & Student D & 400 & 700 & & & & & 400 & & 600 & 400 & & 600 & & 600 & 600 & & 600 & & & 800 & & $50 \%$ \\
\hline 27.40 & Student E & & & 600 & & & 800 & 400 & & & 600 & & & 600 & 800 & 400 & & 400 & & 800 & & & $45 \%$ \\
\hline 27.10 & Student F & 600 & & 700 & & 800 & & 600 & & & & 400 & & 600 & & 400 & & 400 & 600 & & & & $45 \%$ \\
\hline 22.10 & Student $\mathrm{G}$ & 800 & 400 & & 400 & 400 & 600 & & & & 800 & & & & 400 & & & & 600 & 400 & 600 & & $50 \%$ \\
\hline 21.30 & Student $\mathrm{H}$ & & 800 & & & & & & & & & 600 & 400 & 900 & 400 & 400 & 700 & & 600 & & & & $40 \%$ \\
\hline 23.60 & Student I & 400 & & 600 & 600 & & & & & & & 700 & 400 & & 600 & 800 & 400 & 400 & 400 & & & & $50 \%$ \\
\hline 29.10 & Student J & \begin{tabular}{|l|}
600 \\
\end{tabular} & 400 & 400 & 400 & 600 & 400 & 800 & & & & 400 & & & 600 & & & & & 600 & 600 & & $55 \%$ \\
\hline 25.10 & Student K & 400 & 600 & 800 & & & 400 & & 600 & 700 & & & 400 & & & 600 & & & 600 & & & & $45 \%$ \\
\hline 29.50 & Student L & 400 & & & 600 & & & & 400 & 400 & & 400 & & 400 & & 400 & 600 & 800 & & 600 & 600 & & $55 \%$ \\
\hline 26.00 & Student $\mathrm{M}$ & & 600 & 600 & & 600 & 600 & & 900 & & & & 400 & & 400 & 400 & 700 & & & & & & $45 \%$ \\
\hline 22.50 & Student N & & & & 600 & 800 & & 400 & 400 & 600 & 600 & & & 400 & & 600 & 400 & & & 800 & & & $50 \%$ \\
\hline 24.20 & Student O & & & 600 & 600 & 400 & 400 & & 400 & 800 & & 600 & 400 & 400 & 600 & & & 400 & & & & & $55 \%$ \\
\hline 24.00 & Student $\mathrm{P}$ & & 600 & & & & & & 700 & 400 & & & 600 & 700 & 400 & & & 700 & 400 & 600 & & & $45 \%$ \\
\hline 23.70 & Student Q & & & & 600 & 400 & 400 & & & 400 & & & 800 & & & 400 & 700 & & 400 & 600 & 600 & & $50 \%$ \\
\hline 29.40 & Student R & \begin{tabular}{|l|}
600 \\
\end{tabular} & 600 & 400 & & & 600 & 600 & 600 & 400 & & 600 & & & & & 400 & 400 & & 400 & 400 & & $60 \%$ \\
\hline 25.40 & Student S & & & 400 & & 800 & & 400 & & & 600 & & 600 & & 800 & & 600 & 600 & 400 & & 400 & & $50 \%$ \\
\hline \multirow[t]{3}{*}{22.20} & Student $\mathrm{T}$ & 700 & 400 & 400 & 800 & & & 600 & & & 600 & & 600 & & & & & 600 & 400 & 400 & & & $50 \%$ \\
\hline & & & & & & & & & & & & & & & & & & & & & & $\begin{array}{l}\text { Average } \\
\text { Exposure }\end{array}$ & $49 \%$ \\
\hline & & & & & & & & & & & & & & & & & & & & & & SD & $5 \%$ \\
\hline
\end{tabular}

Fig. 12: Frequency of pairs of students being on the same team and the exposure rate of each student as of the end of A400.

\section{Discussion and conclusion}

The methodology presented in this paper is a preliminary approach to potentially more complex decision problems regarding team assignments. Any class size over 10 with more than 3-team requirement is excessive for Excel Solver to find an optimum solution using a single optimization model. Excel Solver does not have the capacity to handle too 
many binary variables at once. The authors of this study were able to overcome this limitation and develop a model for 20 students and 5 teams using a multi-step solver approach. The multi-step approach also allows developing a model that can be updated with the performance of each student throughout the semester, and respond to these updates as building future teams. The model presented in this paper created each team gradually at different times using Excel Solver. The multi-step approach decreased the number of variables considerably as they are defined only for a single team at each step of the optimization. Using this method would allow instructors to get precise results with only the help of Ms. Excel and its Solver add-in, which are widely available for many academic users.

Methodology presented here is kept simple specifically for ease of demonstration. Other users and future research can use additional constraints within the model. Some of these constraints may include the requirements of having at least one person with a certain skill in the group. Certain courses or disciplines may also choose to add constraints to allow for more heterogeneous teams based on minority status, grade history on specific assignments or courses, internship experience, age, etc. Further research on identifying the factors impacting team success and the further development of easy-to-use optimization methodologies will allow programs to improve their outcomes and overall student experience. There is also a need for future studies to compare the performance of student teams that were configured using different methodologies. Finally, future research can focus on converting the spreadsheet methodology introduced here or similar optimization models into executable software programs with easy to use interfaces.

\section{References}

[1] Adams, Stephanie G., and Fernando Pereira, "Measuring faculty preparation to lead teams in the engineering classroom: A pilot study", Proceedings of the American Society for Engineering Education Annual Conference \& Exposition. American Society for Engineering Education, (2002).

[2] Stein, Ruth Federman, and Sandra Hurd, Using student teams in the classroom: a faculty guide, Bolton, MA: Anker Publishing Co., 2000.

[3] Oakley, Barbara, Richard Felder, Rebecca Brent, and Imad Elhajj, "Turning Student Groups into Effective Teams." Journal of Student Centered Learning, Vol. 2, No. 1, (2004), pp. 9-34.

[4] Johnson, D. W., and R. T. Johnson, "Social skills for successful group work. Educational Leadership", Educational Leadership Vol. 47, No. 4, (1989), pp. 29-33.

[5] Chapman, Kenneth J., Matthew Meuter, Dan Toy, and Lauren Wright, "Can't We Pick Our Own Groups? The Influence of Group Selection Method on Group Dynamics and Outcomes", Journal of Management Education Vol. 30, No. 4, (2006), pp. 557-569.

[6] ABET, "Criteria for Accrediting Engineering Programs 2012-2013", (2011), available online http://www.abet.org/DisplayTemplates/DocsHandbook.aspx?id=3143

[7] ACCE, "Standards and criteria for accreditation of postsecondary construction education degree programs", American Council for Construction Education (ACCE) Web site, (2012), available online http://acce-hq.org/documents/DOCUMENT103REVISIONS0712.pdf (accessed January 3, 2013).

[8] Liptak, John J., "Using Emotional Intelligence to Help College Students Succeed in the Workplace", Journal of Employment Counseling, Vol. 42, No. 4, (2005), pp. 171-178.

[9] Schutte, N. S., Malouff, J. M., Bobik, C., Coston, T. D., Greeson, C., Jedlicka, C., et al., "Emotional Intelligence and Interpersonal Relations", The Journal of Social Psychology, Vol. 141, No. 4, (2001), pp. 523-536.

[10] Finelli, Cynthia J., Inger Bergom, and Vilma Mesa, "Student teams in the engineering classroom and beyond: Setting up students for success", CRLT Occasional Papers, No. 29. Ann Arbor, MI: Center for Research on Learning and Teaching, University of Michigan, (2011).

[11] Brickell, J. L., D. B. Porter, M. F. Reynolds, and R. D. Cosgrove, "Assigning Students to Groups for Engineering Design Projects: A Comparison of Five Methods", Journal of Engineering Education, Vol. 83, No. 3, (1994), pp. 259-262.

[12] Bacon, Donald R., Kim A. Stewart, and Elizabeth Scott Anderson, "Methods of Assigning Players to Teams: A Review and Novel Approach", Simulation and Gaming, Vol. 32, No. 1 (2001), pp. 6-17.

[13] Zakarian, Armen, and Andrew Kusiak, "Forming Teams: An Analytical Approach." IIE Transactions on Design and Manufacturing, Vol. 31, No. 1 (1999), 85-97.

[14] Chen, Shi-Jie, and Li Lin, "Modeling Team Member Characteristics for the Formation of a Multifunctional Team in Concurrent Engineering." IEEE Transactions on Engineering Management, Vol. 51, No. 2, (2004), pp. 111-124.

[15] Kirkwood, Craig W. "Selecting student project teams when it really matters: An optimization-based approach using internet resources." Transactions on Education 4, no. 3, (2004), pp. 9-27.

[16] Layton, Richard A., Misty L. Loughry, Matthew W. Ohland, and George D. Ricco, "Design and Validation of a Web-Based System for Assigning Members to Teams Using Instructor-Specified Criteria", Advances in Engineering Education, Vol. 2, No. 1, (2010), pp. 1-28. 